\title{
Optimization of EDX Tomography Acquisition Geometry for Electronic Device Characterization
}

Frieder Baumann $^{1}$, Travis Mitchell ${ }^{1}$, Dirk Utess ${ }^{2}$ and Christian Hobert ${ }^{2}$

${ }^{1}$ GLOBALFOUNDRIES, Inc., Malta, New York, United States, ${ }^{2}$ GLOBALFOUNDRIES, Dresden, Sachsen, Germany

Modern Very Large Scale Integrated (VLSI) circuits consist of devices which are inherently 3D in nature (tri-gate transistors, fin-FETs, NAND memory cells) and which approach nanometer dimensions. Therefore, chemically sensitive tomographic techniques with near atomic resolution have to be developed to characterize these devices and their failure mechanisms [1-3].

In electron tomography, a thin specimen is tilted over a wide range in the electron microscope. Typically, the tilt ranges from $-70^{\circ}$ to $+70^{\circ}$ with data acquisition (images or elemental maps) every 4-5 degrees. However, since projections are missing for the high tilt angles, the reconstruction is not perfect due to the "missing wedge" problem [4].

In this paper, we show how one can improve the data acquisition geometry to mitigate the effect of the missing wedge for certain device features. Since, according to the Center Slice Theorem, each projection represents a central slice of the Fourier-transform of the object, choosing the direction of the tilt axis for a tomogram can mitigate or enhance the effect of the missing information, see fig. 1a). This will be demonstrated using the example of a modern STT-MRAM (Spin Transfer Torque Magnetic Random Access Memory) cell.

Typically, an MRAM cell consists of 15-20 layers of different magnetic and dielectric materials which enable the function of the device [5]. This stack of layers, where each layer is only a few nanometers in thickness, has been characterized using EDX tomography in two different geometries. A 200kV FEI Metrios tool equipped with four windowless SDD detectors was used, the recipe for data acquisition was written using Metrios Recipe Editor [3]. Fig. 1 b) and c) show the tilt axis arrangement and the elemental maps obtained for one of the elements. Although the X-ray signal for the material is strong at every tilt angle, the information content of the set of EDS maps is rather limited due to the "missing wedge". Lack of material or pin holes within the layer can hardly be determined in the current geometry, since the information loss due to the missing wedge is maximized for a plane perpendicular to the tilt axis.

This problem can be alleviated for the MRAM stack by choosing a tilt axis which is not perpendicular to the layers of interest, as shown in Fig. 2 a). The size of the missing wedge is not reduced, but its impact on the layer of interest is greatly diminished. As one can see from fig. 2 b), missing patches of material or other defectivity in the layer, if present, can be observed already in the raw data.

The oblique angle geometry depicted in fig. 2a) allows the full 3D characterization of the experimental MRAM stack as shown in fig. 2c) and d). Only four of the multiple MRAM layers are depicted.

Thus, choosing the optimum geometry for tomography data acquisition of nanometer sized devices can significantly enhance the obtained information and greatly facilitate failure analysis. 

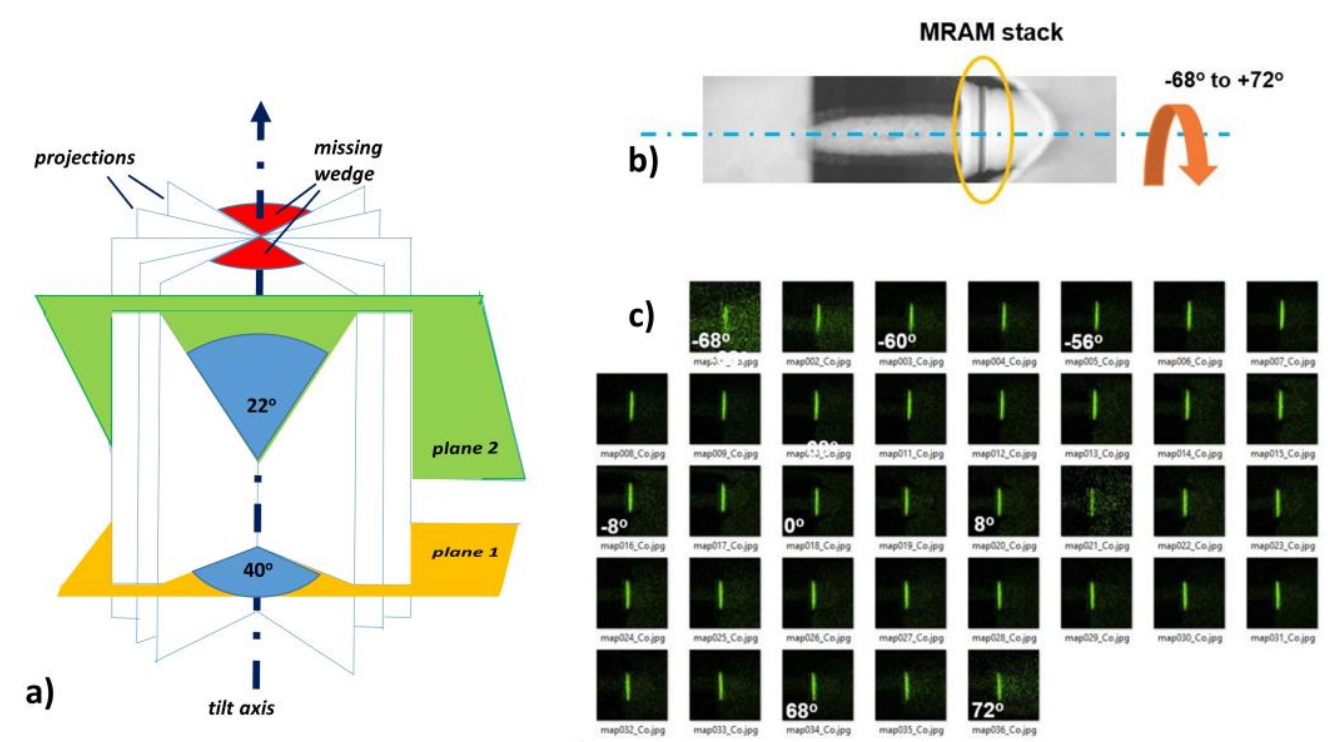

Figure 1. a) Mitigating the effect of the missing wedge. While for object details represented by plane 1 (perpendicular to the tilt axis) the missing wedge amounts to 40o, the "effective missing wedge" for a plane tilted by 350 off the tilt axis (plane 2) amounts to only 22o. : b) EDX tomography arrangement with the tilt axis aligned along the MRAM stack. In this geometry, all layers will be observed edge-on. c) Complete set of EDX maps for one element, Co, obtained for the configuration depicted in (b). Although the EDX signal is strong for each angle, the information content of the map set is limited.
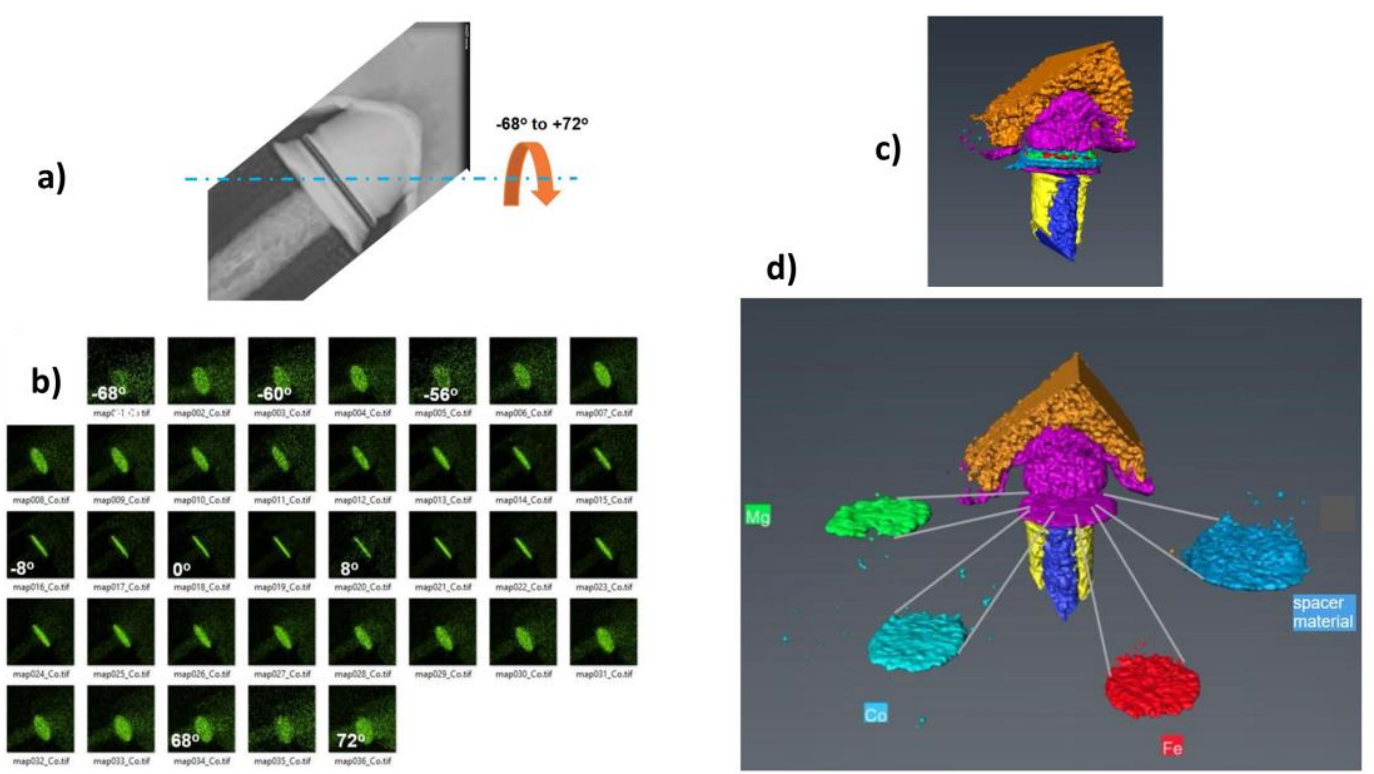

Figure 2. a) EDX tomography experiment with the tilt axis arranged in an oblique angle ( 40o) to the MRAM layers. In this geometry, layers will be mapped not only edge-on, but also partially from top and bottom. This is evident from the complete Co map set depicted in (b). In this geometry, potentially missing patches of material can be observed already in the raw data. c) Fully reconstructed EDX tomography of 
an experimental MRAM cell. d) Same cell as in (c), but several layers "pulled out" to show that each individual layer can be characterized and examined for defects or missing material.

\section{References}

[1] A Genç, et al., Microscopy and Analysis 116, 23-25, 2012

[2] F. H. Baumann et al., Microsc. Microanal. 22 (Suppl 3), 201, 2016

[3] F. H. Baumann et al., Microsc. Microanal. 25 (Suppl 2), 1820, 2019

[4] Electron Tomography, Ed. Joachim Franck, $2^{\text {nd }}$ Edition, Springer, 2006

[5] S. Ikeda et al., IEDM 2014, DOI: 10.1109/IEDM.2014.7047160, 2015 